\title{
The Effect of Turnover Intention on Tie Formation in Online Organization Networks
}

\author{
Wallace Chipidza \\ Claremont Graduate University \\ wallace.chipidza@cgu.edu
}

\author{
John Tripp \\ Clemson University \\ jftripp@clemson.edu
}

\begin{abstract}
Turnover is costly for organizations. While existing research identifies the antecedents and effects of turnover, little research exists on how to identify individuals intending to leave an organization. We hypothesize that individuals with high turnover intention will participate in fewer communication relationships than average, and that individuals prefer communicating with others of similar levels of turnover intention. We use exponential random graph modeling (ERGM) to test our hypotheses on the email and advice networks of a technology company. ERGM allows us to simultaneously examine the effect of individual and dyadic level attributes on network formation. The results support our hypotheses in the email network, but not in the advice network. Our findings imply that organizations should examine their email networks to identify individuals with high turnover intention, and intervene with incentives if they wish to retain the employees.
\end{abstract}

\section{Introduction}

It is increasingly difficult for companies to retain their top talent, particularly in the IT industry. According to a recent LinkedIn report, the software industry exhibits the highest rate of turnover among all industries even above traditionally high turnover industries like restaurants, retail, and hospitality [24]. Turnover of employees often proves costly for organizations. When employees leave companies, they leave with valuable firm- and job-specific knowledge, and also disrupt production processes, delivery of company products, and existing mentor-mentee relationships $[12,30,31]$. Companies not only have to spend resources to hire and train replacements, but they also incur costs from the reduced productivity of replacements relative to established employees. In fact, turnover could cost a company as much as $4 \%$ of its pre-tax annual income [30].

To date, the primary stream of turnover research investigates the factors influencing individual turnover intention. Job satisfaction is the strongest factor influencing turnover intention; the two are negatively related [31, 34]. Other factors include favorable job market, lack of organizational commitment, workplace stress, burnout, and lack of interest in one's job [3, 8, 14]. Extant models of turnover agree that a time lag exists between when an individual first develops high turnover intention to when they actually leave the organization [31]. During this time lag, the individual routinely engages in withdrawal behaviors such as lateness, absenteeism, and withholding effort at work [30]. In this study, we draw on social network theory to argue that social network analysis should reveal individuals engaged in such withdrawal behaviors. Specifically, we argue that individuals with high turnover intention alter their social networking behavior by participating in fewer workplace communication relationships than individuals with low turnover intention. Given that turnover is expensive, companies would benefit if there was a method to identify employees with high turnover intention and potentially intervene before they left. Our study proposes such a method.

Existing research shows that multiple advantages accrue to individuals occupying highly central positions in organizational networks. Such advantages include high job satisfaction, high perceived job security, and better job performance [37, 39]. Some advantages are associated with online networks, others with offline networks, and others with both [1, 33, 37, 39]. To date, however, little to no research has been done to examine whether co-occurring online and offline networks wield disparate influences on individual work outcomes. This study addresses that shortcoming within the literature on organizational social networks.

As a burgeoning field, research in social networks offers many possibilities for understanding the interconnected nature of human interactions. One prominent feature of existing research is the focus on the relationship between individual centrality and various important outcomes. Centrality primarily varies because individuals have varying numbers of 
connections, i.e. some people are more popular than others, and because individuals occupy varying positions in the network, i.e. some are deeply embedded in the middle of the network, while others occupy the fringes. Techniques for calculating such centralities have existed for decades; thus, it has been fairly easy for researchers to investigate the effects of centrality on many variables using frequentist techniques such as multiple regression, ANOVA, and structural equation modeling [37, 39]. The use of centrality to underscore the importance of networks is thus understandable.

However, emphasizing centrality in social network research leaves out a key part of what networks entail. Centrality is an individual level construct, to the extent that each individual in a network possesses some score that indicates their influence within the network. Yet social networks are not only about individuals (nodes), but are also about relationships (ties or edges). Our study is in part motivated by the observation that research on social network relationships is rare, relative to research on individuals. A relationship is a link between two individuals [36]. If one considers the relationship and the attributes of the individuals linked by that relationship, they are concerned with the dyad. In this study, we ask the following research question:

\section{$R Q:$ How does turnover intention influence tie formation within an online organizational social network?}

To answer our research question, we explore whether individuals with similar levels of turnover intention tend to have relationships within organizations, and whether this effect is consistent across offline and online networks. We also examine whether the number of relationships an individual participates in varies according to their turnover intention. We employ email network and advice network data collected during the same period from employees in a technology organization and analyze it using exponential random graph modeling (ERGM) [28]. Our method of choice is particularly useful because it allows for the examination of complex models of tie formation that include multiple factors at multiple levels of analysis while controlling for confounds. To the best of our knowledge, ours is the first study to examine how individuals engage in networking behavior based on their turnover intention. In the next section, we describe related research and identify where our study fits in the ecosystem of social network research.

\section{Related Research}

\subsection{Social networks}

A social network is a set of entities and their relationships [36]. Entities are social actors or their groupings, i.e. individuals, teams, organizations, and communities; in social network lexicon they are referred to as nodes. Relationships between nodes are referred to as ties or edges. Sometimes network relationships are directional, sometimes not. For example, on Twitter person A may follow person B while B does not follow A. Other times relationships do not have direction as is the case with Facebook friendship ties.

2.1.1. Antecedents of tie formation in social networks. Tie formation in social networks has been theorized to depend on two broad categories of factors - structural and demographic. Structural factors are endogenous network mechanisms that guide the creation of new ties and the maintenance of existing ties; in other words, the current state of the network is dependent on the previous state of the network [6]. Demographic factors capture the influences of exogenous attributes and are not influenced by network structure [19].

The differences among structural and demographic factors can be understood by reviewing how they are calculated. To calculate whether a structural factor is significant in tie formation, one does not need extra information about the nodes except their identities and ties among them. Hence, to calculate the structural preference for isolation in the network for example, one would count the number of nodes with at least one tie and the number of nodes with zero ties; if these numbers deviate from what should be expected from chance, the network will display a positive or negative tendency towards tie formation. On the other hand, to calculate the effect of demographic factors, one requires knowledge of exogenous attributes like age, gender, turnover intention, etc. and these are independent of network structure.

To date, most social network research focuses on the effects of social network centrality on various outcomes - most of them positive. For example, high betweenness centrality is associated with greater work performance and higher compensation [9, 37]. Research on network formation is relatively rare [7, 38]. Where it has been done, such research emphasizes individual, single-item attributes such as gender, age, and income and their influence on tie formation. Our study examines how homophily according to turnover intention influences tie formation within organizational social networks, and whether the strength and direction of those influences vary across online vs. offline networks. In the process, our 
analyses help identify whether individuals with high turnover intention exhibit different networking behavior in online and offline contexts.

\subsection{Turnover intention}

Turnover intention - defined as "a conscious and deliberate willingness to leave the organization" [12:286] - is the strongest predictor of actual turnover in organizations. It is negatively related with job satisfaction and organizational commitment [3, 31]. Turnover can be functional or dysfunctional; while functional turnover is considered desirable and occurs when poor job performers leave the organization, dysfunctional turnover is undesirable because it occurs when high performers leave [29]. This imposes various costs on the organization including financial cost and disruption of production and delivery processes [5, 12]. Existing research also identifies various mechanisms for reducing dysfunctional turnover, including increased compensation for highperforming employees and the availability of channels for employees to air their grievances [29, 32]. Thus, there is extensive literature on the effects of turnover and how to potentially reduce it. What is lacking in the research are mechanisms to identify employees with high turnover intention, and below we present a theoretical explanation for the link between an individual's social networking behavior and her turnover intention.

\section{Theoretical Model}

Employees with high turnover intention engage in withdrawal behaviors such as absenteeism, lateness, and withholding work effort [30]. It is feasible that such withdrawal behaviors imply changes in social networking behaviors. Employees intending to leave the organization are less motivated to work towards achieving organizational goals than those willing to stay [5]. We expect that individuals wishing to leave the organization may also weaken or dissolve their existing ties with their workmates. In an email network, these changes are likely to manifest as reduced outgoing communication with others both from ignoring emails and from not initiating email conversations with others.

Moreover, because each employee is embedded within formal and informal social networks at work, it is possible that turnover intention may also be a function of their ties with others [31]. In other words, we are likely to observe homophily according to turnover intention for two reasons, dubbed selection and socialization. In the selection mechanism, because "birds of a feather flock together," individuals select others with similar levels of turnover intention to form ties with $[11,26]$. In the socialization mechanism, individuals are likely to be influenced by the attitudes held by their close connections; thus, one's level of turnover intention may influence the turnover intention of close work colleagues [22]. Although selection and socialization are difficult to disentangle with cross-sectional studies, they both result in observable homophily. In this study, we examine a model of network formation that includes (I) variation in tie forming behavior and (II) homophily according to turnover intention. Figure 1 portrays this model.

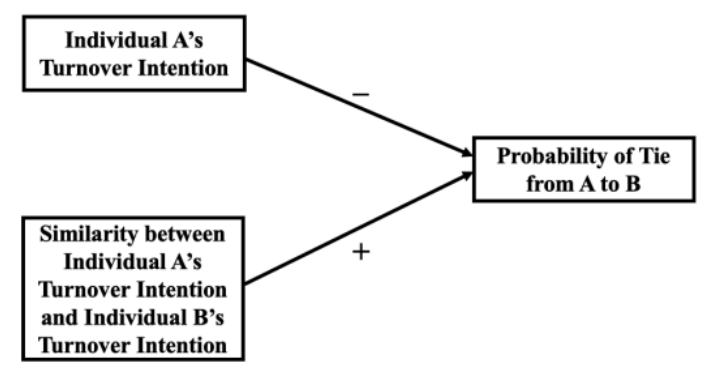

Figure 1: Model of tie formation according to turnover intention

We present evidence that employees with high turnover intention exhibit different networking behavior than employees with low turnover intention, and these behaviors vary across online and offline networks.

\section{Method}

\subsection{Data Collection}

We collected our data from TechCo (a pseudonym), a technology firm based in Singapore which employs 50 people. We used metadata from TechCo's email logs to construct its internal email network. The metadata for an email includes the identities of the sender and receiver(s) of the email. The email data is pseudonymized to protect employee privacy. An outgoing tie exists from node A to B if A sends a number of emails exceeding the mean number of emails sent from each individual in the relevant period. Dichotomizing ties in this way is necessary for our model assessment technique, and is common in studies utilizing the social networks perspective $[17$, 33].

To assess levels of turnover intention, we administered an anonymous survey to TechCo employees with the following questions and statements drawn from [25]: 
1. I will be with this company five years from now.

2. How likely is it that you will be working with your current company this time next year?

3. I will probably look for a job at a different company in the next year.

4. How likely is it that you will take steps during the next year to secure a job at a different company?

We surveyed the employees on the people they most seek work-related advice from, to build the offline advice network. We presented the following statement - drawn from [10] - to solicit information on advice ties:

Indicate the extent to which you turn to each of the following people for expert advice about work-related activities.

We had 42 respondents out of 50 potential respondents, for a response rate of $84 \%$ which exceeds the threshold of $80 \%$ required for empirical social networks research [39]. The responses from the survey were not shared with the organization, to protect employee privacy. Data collection took place during the month of August 2017.

Estimating the parameters capturing the relationships in the model is not possible using generalized linear regression, because social network data violates the required independence of observations assumption. What is needed is a technique that also models the various dependencies among nodes in the network, e.g., the homophily effect of turnover intention outlined above. A technique called exponential random graph modeling (ERGM) has such capabilities, and is outlined in detail next.

\subsection{Exponential random graph modeling (ERGM).}

While social networks exhibit some randomness in the formation of ties, they also exhibit certain nonrandom tendencies. The overall goal of ERGM is to describe, with statistical confidence, both the significance and relative strengths of these forces that shape a given social network [28]. Such forces may be structural or endogenous, meaning that they are properties of the overall network, or demographic or exogenous, meaning that they are derived from the influences of node attributes. Structural forces include the propensity for tie formation, measured by density, and exogenous forces include homophily and heterophily.
4.2.1. ERGM parameter estimation procedure. To arrive at a statistical determination of the significances of both structural and exogenous effects, a series of derivations must be outlined. First, consider a network with a given size or number of nodes, $n$. There is an exponential number, with respect to $n$, of possible networks. Specifically, an observed network of size $n$ is one of $2 n$ possible networks with the same size. Given the set of all these random networks, what is the probability of the observed network?

Theoretical and empirical evidence from past research shows that not all networks in the set of random networks are equally likely. For example, real world networks typically show homophily, i.e. nodes in a social network typically prefer forming ties with similar others [4, 26], and bi-directed networks typically show reciprocity, i.e., if a tie exists from node A to $\mathrm{B}$, it is highly likely that the reverse tie exists [13]. As such, a network is likely to include several nonrandom effects that have varying degrees of strength. ERGM expresses the conditional probability of the observed network given the random set of networks as the value of all the relevant weighted effects divided by the summed value of all the possible networks:

$$
\mathrm{P}(\mathrm{Y}=\mathrm{y})=\frac{\exp \left\{\theta^{\mathrm{T}} \mathrm{z}(\mathrm{y})\right\}}{\kappa(\theta, \mathrm{Y})}, \mathrm{y} \in \mathrm{Y}
$$

where $Y$ is the random variable of all possible networks of the same size of the observed network, $y$ is the observed network. The numerator is an exponent of $z(y)-$ the set of model effects for the observed network $y$-multiplied by the vector of their associated weights $\theta^{T}$. The denominator is the summed value of all possible networks, multiplied by a normalizing constant to ensure that the total probability equals 1 [28]. For very small networks, the denominator can be easily calculated. However, as the number of nodes in the network rises, the number of possible networks increases exponentially. Hence, calculating the values for all possible networks becomes infeasible.

To estimate the denominator, Markov Chain Monte Carlo (MCMC) methods are employed. MCMC extracts a sample of networks that follows the probability distribution of all the possible networks. The algorithm generates a sequence of networks by adding or removing a single tie, such that each network in the sequence depends only on the previous network i.e. a Markov Chain [35]. At each step, the probability of the generated network is calculated; the new network is retained only if its probability exceeds that of the previous network i.e. a Monte Carlo method because the procedure uses randomization to perform a computation in fixed time, but with uncertain output 
[20].

For practical utility, instead of obtaining the probability of the network, it is more useful to reexpress the equation so that we obtain the probability of a tie, conditional on the network. This is analogous to binary logistic regression, in that we obtain the probability of a tie, given the structural and node properties of the participating nodes. The goal is to find the thetas, or parameter estimates that would maximize the probability of the observed network. Reexpressed, the conditional log-odds of a tie are as follows:

$$
\operatorname{logit}\left(P\left(Y_{i j}=1 \mid n, Y_{i j}^{c}\right)\right)=\sum_{k=1}^{K} \theta_{k} \delta z_{k}(y)
$$

where $Y_{i j}=1$ indicates the presence of a tie from node $i$ to node $j, \mathrm{Y}_{\mathrm{ij}}^{\mathrm{c}}$ are all the other dyads in the network, the expression $\delta z_{k}(y)$ is the amount by which $z_{k}(y)$ changes as a result of switching $Y_{i j}$ from 0 to 1 . Because tie formation between any two nodes may not be independent, the probability of any tie is conditional on the configuration of other ties in the network, hence the inclusion of the $Y_{i j}^{c}$ term.

We used the $R$ statnet package to fit ERGMs onto our email and advice networks [20,21]. The algorithm proceeds as follows: first guess $\theta_{k}$ using maximum pseudolikelihood estimation (MPLE). Using MPLE assumes that dyads are independent, hence it is only used to obtain an initial guess of the vector of thetas, which is likely to be inaccurate. Second, simulate a set of random networks using the guess from step 1 . Third, use the simulated sample to find a better $\theta_{k}$ using maximum likelihood estimation. Fourth, iterate steps 2 and 3 until the simulated network is similar to the observed network - at this point the algorithm would have converged and reliable estimates of $\theta_{k}$ would have been obtained.

4.2.2. Model specification. We compare the relative strengths of the forces shaping tie formation in TechCo's email and advice networks. These forces are: the tendency for tie formation, reciprocity of ties, the homophily effect according to turnover intention, and the covariate effect of turnover intention. While the homophily effect of turnover intention captures the extent to which individuals with similar levels of turnover intention prefer to form ties with each other, the covariate effect of turnover intention captures the relationship between turnover intention and the number of ties possessed by an individual in the network. We control for the covariate effect of the importance of email in conducting work.

To understand the effects of structural and node attributes on network formation, we first create a baseline model that calculates the probability of a tie the null model, which counts the number of ties in the network. We use the statnet package in $\mathrm{R}[16,20]$ to specify our model. The number of ties of the network forms the basis of the null model. We also add the reciprocity - a structural attribute - to the model. Reciprocity is the extent to which pairs of nodes form mutual connections with each other [13], and is a general feature of directed networks [36].

Next, we add the node attribute influences to the model, beginning with (I) variation in number of outgoing ties, and (II) homophily according to turnover intention to the model. Recall that a tie exists from node A to node B whenever A sends B a number of emails that exceeds the organization's mean in the specified time period. We measure II as the absolute difference between the turnover intention levels of pairs of nodes in the network. We also add the variation in the number of incoming ties to the model to capture any variations in the number of incoming ties according to turnover intention. We control for the covariate effect of the importance of email use to an individual's everyday work activities. The resulting model is compared to the baseline model to assess whether there is an improvement using the Akaike Information Criterion (AIC) [2, 18]. The AIC is a method for comparing models, and the smaller its result the better the model. Table 1 summarizes these terms and their definitions.

Table 1: Model terms and definitions

\begin{tabular}{|c|c|}
\hline Term & Description \\
\hline Density & Number of ties [20] \\
\hline Reciprocity & $\begin{array}{c}\text { Number of pairs of nodes with } \\
\text { bidirectional ties [20] }\end{array}$ \\
\hline $\begin{array}{c}\text { Homophily } \\
\text { (heterophily) }\end{array}$ & $\begin{array}{c}\text { Sum of absolute differences of an } \\
\text { attribute for every node pair with a } \\
\text { tie [20] }\end{array}$ \\
\hline $\begin{array}{c}\text { Covariate } \\
\text { effect } \\
\text { continuous } \\
\text { variable) }\end{array}$ & $\begin{array}{c}\text { Sum of values of an attribute for } \\
\text { every node pair with a tie [15] }\end{array}$ \\
\hline
\end{tabular}

\section{Results}

\subsection{Descriptive results and network plots}

The gender composition of the respondents to our survey was $32 \%$ female and $68 \%$ male. The average turnover intention was 3.04 (on a scale of $1-7$ ), with a standard deviation of 1.34. The density of the email network was low relative to the advice networks 
(Table 2).

Table 2: Network characteristics of email vs advice networks

\begin{tabular}{|l|l|l|l|}
\hline Network & $\begin{array}{l}\text { Number } \\
\text { of } \\
\text { Nodes }\end{array}$ & $\begin{array}{l}\text { Number } \\
\text { of Ties }\end{array}$ & Density \\
\hline Email & 41 & 258 & 0.16 \\
\hline Advice & 41 & 307 & 0.18 \\
\hline
\end{tabular}

Figures 2 and 3 are plots of the email and advice networks at TechCo respectively, with the color of the node representing its level of turnover intention.

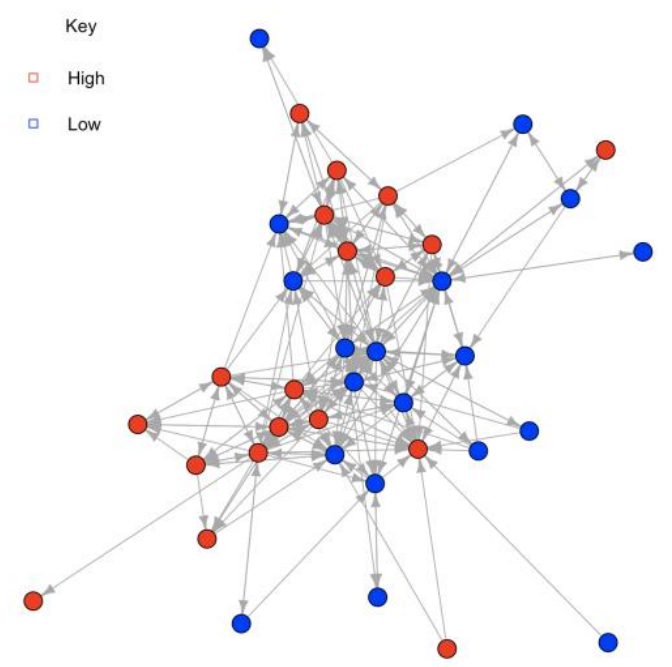

Figure 2: Email network for TechCo, with nodes colored by level of turnover intention

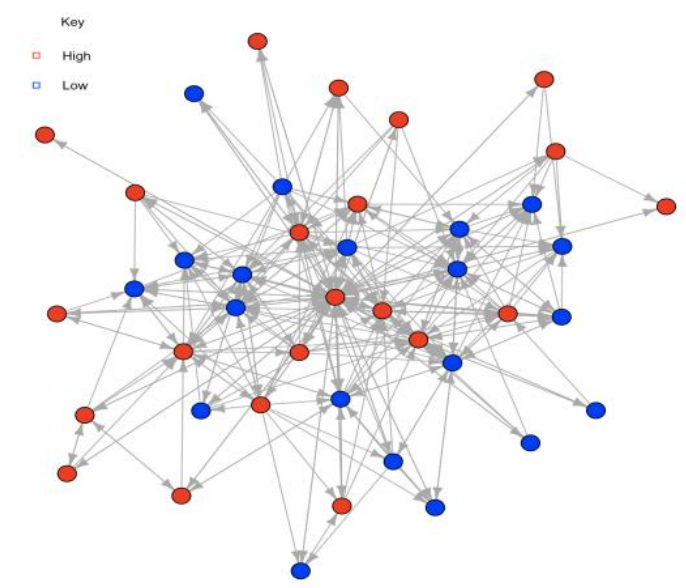

Figure 3: Advice network for TechCo, with nodes colored by level of turnover intention
Several observations are evident from the email and advice plots above. It would seem that the most central individuals by number of incoming ties i.e., those deeply embedded within the email network generally have low levels of turnover intention. The situation seems different in the advice network, which might mean that individuals from whom advice is most frequently solicited are likely to have high levels of turnover intention. Next, we present our results from modeling network formation using ERGM.

\subsection{Results from exponential random graph modeling (ERGM)}

As is standard in using ERGM, we create a baseline model of network formation using only the density term. This model can be understood as a null hypothesis which states that all the ties in the network randomly arose. The results of fitting the baseline model onto the email network are presented in Table 3 . The negative edges parameter indicates that there is a negative tendency to form ties within the network and this is typical in real-world networks. Relative to the maximum number of ties possible of 1640 within a 41-node bidirectional network, the network only has 258 edges.

Table 3: Results of fitting baseline model to observed email network

\begin{tabular}{|l|l|c|c|}
\hline & $\begin{array}{l}\text { Parameter } \\
\text { Estimate }\end{array}$ & $\begin{array}{c}\text { Std. } \\
\text { Error }\end{array}$ & p-value \\
\hline Edges & -1.49 & 0.07 & 0.00 \\
\hline
\end{tabular}

$\mathrm{AIC}=1342$

Our hypothesized model postulates that the email network (shown in Figure 2) arises because of reciprocity, variations in turnover intention, and homophily according to turnover intention. Individuals with low levels of turnover intention are hypothesized to form more ties than individuals with high levels of turnover intention. We create a model that incorporates baseline density, reciprocity, the main and homophily effects of turnover intention, and the control effect of email's importance to one's work. Results of fitting this model onto the email network are shown in Table 4.

The AIC for the hypothesized model (1078) is lower than that of the baseline model (1342), which means that the hypothesized model is an improvement on the baseline model. Each parameter estimate is the log-odds of a tie between two nodes, conditional on the rest of the network [18]. The parameter for edges is non-significant, meaning that the conditional logodds of a tie is zero, for a tie probability of 0.5 . The 
parameter for reciprocity is positive and is the strongest, indicating that the email network is marked by high reciprocity. Because the parameter is a conditional log-odds, it can be converted to a probability using $1 /(1+e$-estimate), which evaluates to 0.97 . This means that there is a $97 \%$ probability that a tie will be formed from B to A, if A to B exists. And if there is a 1-unit difference between the turnover intentions of $\mathrm{A}$ and $\mathrm{B}$, the probability that they will form a tie decreases by $3 \%$. Moreover, an individual who increases their turnover intention by one unit decreases their probability of forming a new tie by $3.2 \%$.

Table 4: Results of fitting structural and node attribute model to observed email network

\begin{tabular}{|l|c|l|l|}
\hline & $\begin{array}{l}\text { Parameter } \\
\text { Estimate }\end{array}$ & $\begin{array}{l}\text { Std. } \\
\text { Error }\end{array}$ & $\begin{array}{l}\text { p- } \\
\text { value }\end{array}$ \\
\hline Edges & -0.55 & 0.51 & 0.27 \\
\hline Reciprocity & 3.60 & 0.27 & 0.00 \\
\hline $\begin{array}{l}\text { Homophily } \\
\text { (Turnover } \\
\text { Intention) }\end{array}$ & 0.13 & 0.06 & 0.02 \\
\hline $\begin{array}{l}\text { Outgoing ties } \\
\text { (Turnover } \\
\text { Intention) }\end{array}$ & -0.13 & 0.07 & 0.07 \\
\hline $\begin{array}{l}\text { Incoming ties } \\
\text { (Turnover } \\
\text { Intention) }\end{array}$ & 0.21 & 0.07 & 0.00 \\
\hline $\begin{array}{l}\text { Outgoing Ties } \\
\text { (Email } \\
\text { Importance) }\end{array}$ & -0.31 & 0.12 & 0.01 \\
\hline $\begin{array}{l}\text { Incoming ties } \\
\text { (Email } \\
\text { Importance) }\end{array}$ & -0.20 & 0.12 & 0.10 \\
\hline $\begin{array}{l}\text { AmC 1078 } \\
\text { Ampoing }\end{array}$ & & & \\
\hline
\end{tabular}

$$
\mathrm{AIC}=1078
$$

Tables 5 and 6 show the results of fitting the baseline and hypothesized models onto the advice network. As with the email network, the hypothesized model better explains the observed network than the baseline model, as shown by the decrease in AIC from 1616 to 1431 after adding structural and node attribute terms to the model. Reciprocity is also the strongest force shaping the advice network, meaning that individuals are likely to seek advice from those that seek advice from them. However, we observe no homophily according to turnover intention, and there is no difference in outgoing tie-forming behavior as an individual's turnover intention varies. A possible explanation for high levels of turnover intention among popular individuals in the advice network is that they may be valued for their expertise and thus have more favorable opportunities to move elsewhere. Such individuals might also feel exhausted from having to continuously give work-related advice to their colleagues, leading them to seek other opportunities.

Table 5: Results of fitting baseline model to observed advice network

\begin{tabular}{|l|c|c|c|}
\hline & $\begin{array}{c}\text { Parameter } \\
\text { Estimate }\end{array}$ & $\begin{array}{c}\text { Std. } \\
\text { Error }\end{array}$ & p-value \\
\hline Edges & -1.53 & 0.06 & 0.00 \\
\hline \multicolumn{2}{|l}{ AIC $=1616$}
\end{tabular}

Table 6: Results of fitting structural and node attribute model to observed advice network

\begin{tabular}{|l|c|l|l|}
\hline & $\begin{array}{l}\text { Parameter } \\
\text { Estimate }\end{array}$ & $\begin{array}{l}\text { Std. } \\
\text { Error }\end{array}$ & $\begin{array}{l}\text { p- } \\
\text { value }\end{array}$ \\
\hline Edges & -2.14 & 0.49 & 0.00 \\
\hline Reciprocity & 2.85 & 0.21 & 0.00 \\
\hline $\begin{array}{l}\text { Homophily } \\
\text { (Turnover } \\
\text { Intention) }\end{array}$ & -0.03 & 0.05 & 0.52 \\
\hline $\begin{array}{l}\text { Outgoing ties } \\
\text { (Turnover } \\
\text { Intention) }\end{array}$ & -0.06 & 0.05 & 0.25 \\
\hline $\begin{array}{l}\text { Incoming ties } \\
\text { (Turnover } \\
\text { Intention) }\end{array}$ & 0.13 & 0.06 & 0.02 \\
\hline $\begin{array}{l}\text { Outgoing ties } \\
\text { (Email } \\
\text { Importance) }\end{array}$ & -0.19 & 0.10 & 0.07 \\
\hline $\begin{array}{l}\text { Incoming ties } \\
\text { (Email } \\
\text { Importance) }\end{array}$ & 0.07 & 0.10 & 0.51 \\
\hline \multicolumn{1}{|l|}{ AIC =1431 } & & \\
\hline
\end{tabular}

5.2.1. Goodness of fit for ERGM. To assess goodness of fit, we first generate 100 networks using the parameter estimates obtained by running ERGM on the observed network; 100 is adequate for the test [19]. From the simulated set of networks, we obtain probability distributions of the terms included in the model; the values obtained in the observed network are then compared to the values in the simulated networks. If there are no significant differences among these values, it can be concluded that the model has sufficient goodness of fit. The probability distributions of the statistics included in the hypothesized model do not differ between the email and advice networks and their corresponding sets of simulated networks. 


\section{Discussion}

The extensive literature on turnover states that individuals with strong turnover intentions engage in withdrawal behaviors such as lateness, absenteeism, and withholding effort from work [30]. Based on these findings, we hypothesized that such individuals are also likely to reduce their levels of participation in organization social network relationships. We also hypothesized that individuals prefer forming ties with others of similar turnover intention through the selection and socialization mechanisms that constitute homophily [22].

The results from fitting ERGMs on the email network support our hypotheses. First, we find that individuals are more likely to have email communication ties with workmates that have similar levels of turnover intention than with those with dissimilar turnover intention. We cannot determine whether this homophily arises out of selection or socialization. Individuals might select to communicate with others with similar levels of turnover, or they might influence their close contacts within the organization to develop high levels of turnover intention. Individuals with high turnover intention may share their negative workplace experiences with their close contacts, or they may inform them of better job opportunities, thus influencing their counterparts to develop strong turnover intentions.

Second, we find that individuals with high turnover intention participate in fewer email communication relationships than individuals with low turnover intention. When individuals intend to leave an organization, they are less committed to the success of the organization, or they may develop negative attitudes towards the organization $[29,31]$. Thus, such individuals are less motivated to respond to emails or to initiate new conversations with their workmates. Indeed, we find that individuals with high turnover intention tend to have higher numbers of incoming email ties than average, which suggests that they may be ignoring emails from their work colleagues.

Third, a large body of research exists on the effects of social network centrality on outcomes like job satisfaction, perceived job security, and organizational commitment [e.g. 27, 39]. Our study is a rare exception in that we examine the effect of a psychological construct, i.e. turnover intention on social networking behavior. Our study is also to be contrasted with extant research because it shows that the effect of turnover intention on networking behavior varies across online vs. offline networks. This variation offers potential of interesting further research.
It is notable that we did not observe variation in tieforming behavior and homophily according to turnover intention in the advice network. To generate the advice network, we use self-reported data from TechCo employees. Self-reported data suffers from many limitations including inaccurate recall and desirability bias [23]. Nevertheless, we observed that individuals with high turnover intention tend to have higher numbers of incoming advice ties on average. This might suggest that the individuals valued for their expertise may have more favorable outside job opportunities, and may thus be motivated to move. Such individuals might also feel that having to continuously assist others undermines their own work, and thus desire to seek other opportunities. Further research is required to better understand these findings.

Taken together, our findings imply that the email network reveals useful information about turnover intentions. An organization that tracks changes in its email network may be able to identify those with high turnover intention by looking for withdrawal behaviors such as ignoring emails and not initiating as many new conversations. If these high turnover intention individuals are high performers, management may intervene by offering incentives for them to stay and save money and resources in the process. Research on dysfunctional turnover shows that individualized performance incentives are more effective at retaining high performance individuals than group incentives [32]. Examining the email network helps the organization identify high turnover intention individuals, which informs personalized intervention.

Our study has limitations. One limitation is that our data was collected in one organization that has its own distinctive culture and characteristics. Future research could examine organizations of different sizes and industries. Lack of access to email content also forms a limitation of the current study. Examining the email content would add better understanding of the link between social networking behavior and turnover intention in organizations. Further, other variables such as rank and expertise should be added to the model to determine whether they influence tieforming behavior in social networks. Another limitation is that other informal networks, e.g., friendship and trust networks may better capture the influence of turnover intention on tie forming behavior. Overcoming these limitations offers potentially fruitful opportunities for future research.

\section{Conclusion}


In this study, we examined how individuals vary their tie-forming behaviors according to turnover intention. We hypothesized that as turnover intention increases, individuals will maintain fewer outgoing ties with their work colleagues. We also hypothesized that as the difference in turnover intentions between two individuals decreases, the probability that they will form a tie increases. Using exponential random graph modeling, we found support for our hypotheses in the email network, but not in the advice network. Our findings suggest that the email network may indirectly reveal information about turnover intention of an organization's employees. Organizations may find it useful to use email network data to identify those employees intending to leave, and intervene with incentives if they so desire.

\section{References}

[1] Agneessens, F., and R. Wittek, "Social capital and employee well-being: disentangling intrapersonal and interpersonal selection and influence mechanisms", Revue française de sociologie 49(3), 2008, pp. 613637.

[2] Akaike, H., “A new look at the statistical model identification", Automatic Control, IEEE

Transactions on 19(6), 1974, pp. 716-723.

[3] Allen, N.J., and J.P. Meyer, "The measurement and antecedents of affective, continuance and normative commitment to the organization", Journal of occupational psychology 63(1), 1990, pp. 1-18.

[4] Barabási, A.-L., and E. Bonabeau, "Scale-free", Scientific American, 2003.

[5] Beecroft, P.C., F. Dorey, and M. Wenten, "Turnover intention in new graduate nurses: a multivariate analysis", Journal of advanced nursing 62(1), 2008, pp. 41-52.

[6] Block, P., "Reciprocity, transitivity, and the mysterious three-cycle", Social Networks 40, 2015, pp. 163-173.

[7] Borgatti, S.P., A. Mehra, D.J. Brass, and G. Labianca, "Network analysis in the social sciences", Science 323(5916), 2009, pp. 892-895.

[8] Brooke Jr, P.P., and J.L. Price, "The determinants of employee absenteeism: An empirical test of a causal model", Journal of Occupational Psychology 62(1), 1989, pp. 1-19.
[9] Burt, R.S., "The social capital of structural holes", The new economic sociology: Developments in an emerging field, 2002, pp. 148-190.

[10] Cross, R., S.P. Borgatti, and A. Parker, "Beyond answers: dimensions of the advice network", Social networks 23(3), 2001, pp. 215-235.

[11] Currarini, S., M.O. Jackson, and P. Pin, “An economic model of friendship: Homophily, minorities, and segregation", Econometrica 77(4), 2009, pp. 1003-1045.

[12] Egan, T.M., B. Yang, and K.R. Bartlett, "The effects of organizational learning culture and job satisfaction on motivation to transfer learning and turnover intention", Human resource development quarterly 15(3), 2004, pp. 279-301.

[13] Garlaschelli, D., and M.I. Loffredo, "Patterns of link reciprocity in directed networks", Physical review letters 93(26), 2004, pp. 268701.

[14] Gellatly, I.R., "Individual and group determinants of employee absenteeism: Test of a causal model", Journal of organizational behavior 16(5), 1995, pp. 469-485.

[15] Goodreau, S.M., J.A. Kitts, and M. Morris, "Birds of a feather, or friend of a friend? Using exponential random graph models to investigate adolescent social networks", Demography 46(1), 2009, pp. 103-125.

[16] Handcock, M.S., D.R. Hunter, C.T. Butts, S.M. Goodreau, and M. Morris, "statnet: Software tools for the representation, visualization, analysis and simulation of network data", Journal of statistical software 24(1), 2008, pp. 1548.

[17] Hanneman, R.A., and M. Riddle, Introduction to social network methods, University of California Riverside, 2005.

[18] Harris, J.K., An introduction to exponential random graph modeling, Sage Publications, 2013.

[19] Hunter, D.R., S.M. Goodreau, and M.S. Handcock, "Goodness of Fit of Social Network Models", Journal of the American Statistical Association 103(481), 2008, pp. 248-258.

[20] Hunter, D.R., M.S. Handcock, C.T. Butts, S.M. Goodreau, and M. Morris, "ergm: A package to fit, simulate and diagnose exponential-family models for 
networks", Journal of statistical software 24(3), 2008, pp. nihpa54860.

[21] Ihaka, R., and R. Gentleman, "R: a language for data analysis and graphics", Journal of computational and graphical statistics 5(3), 1996, pp. 299-314.

[22] Kandel, D.B., "Homophily, selection, and socialization in adolescent friendships", American journal of Sociology 84(2), 1978, pp. 427-436.

[23] Kane, G.C., M. Alavi, G.J. Labianca, and S. Borgatti, "What's different about social media networks? A framework and research agenda", MIS Quarterly, 2012.

[24] Linkedin, These 3 Industries Have the Highest Talent Turnover Rates, 2018.

[25] McKnight, D.H., B. Phillips, and B.C. Hardgrave, "Which reduces IT turnover intention the most: Workplace characteristics or job characteristics?", Information \& Management 46(3), 2009, pp. 167-174.

[26] McPherson, M., L. Smith-Lovin, and J.M. Cook, "Birds of a feather: Homophily in social networks", Annual review of sociology, 2001, pp. 415-444.

[27] Merten, F., and P. Gloor, "Too much e-mail decreases job satisfaction", Procedia-Social and Behavioral Sciences 2(4), 2010, pp. 6457-6465.

[28] Morris, M., M.S. Handcock, and D.R. Hunter, "Specification of Exponential-Family Random Graph Models: Terms and Computational Aspects", Journal of statistical software 24(4), 2008, pp. 1548-7660.

[29] Park, H.Y., J. Ofori-Dankwa, and D.R. Bishop, "Organizational and environmental determinants of functional and dysfunctional turnover: Practical and research implications", Human Relations 47(3), 1994, pp. 353-366.

[30] Sagie, A., A. Birati, and A. Tziner, “Assessing the costs of behavioral and psychological withdrawal: A new model and an empirical illustration", Applied psychology 5l(1), 2002, pp. 67-89.

[31] Soltis, S.M., F. Agneessens, Z. Sasovova, and G. Labianca, "A social network perspective on turnover intentions: The role of distributive justice and social support”, Human Resource Management 52(4), 2013, pp. 561-584.
[32] Sturman, M.C., C.O. Trevor, J.W. Boudreau, and B. Gerhart, "Is it worth it to win the talent war? Evaluating the utility of performance-based pay", Personnel Psychology 56(4), 2003, pp. 997-1035.

[33] Sykes, T.A., V. Venkatesh, and S. Gosain, "Model of acceptance with peer support: A social network perspective to understand employees' system use", MIS quarterly, 2009, pp. 371-393.

[34] Trevor, C.O., "Interactions among actual easeof-movement determinants and job satisfaction in the prediction of voluntary turnover", Academy of management journal 44(4), 2001, pp. 621-638.

[35] Van Der Pol, J., "Introduction to network modeling using Exponential Random Graph models (ERGM)", 2016.

[36] Wasserman, S., and K. Faust, Social network analysis: Methods and applications, Cambridge university press, 1994.

[37] Wu, L., "Social network effects on productivity and job security: Evidence from the adoption of a social networking tool", Information systems research 24(1), 2013, pp. 30-51.

[38] Yan, L. (Lucy), J. Peng, and Y. Tan, "Network Dynamics: How Can We Find Patients Like Us?", Information Systems Research 26(3), 2015, pp. 496512.

[39] Zhang, X., and V. Venkatesh, "Explaining Employee Job Performance: The Role of Online and Offline Workplace Communication Networks.", Mis Quarterly 37(3), 2013, pp. 695-722. 